\title{
Zur Konkurrenz der Impfstoffe gegen COVID-19: Aufklärung und Auswahl
}

\section{Dieter Hart}

\section{Einführung}

Wegen bestehender Knappheit und organisatorischen Herausforderungen hat sich die Vorsitzende des Ethikrates gegenwärtig gegen eine „freie Wahlmöglichkeit“ des Corona-Impfstoffs ausgesprochen ${ }^{1}$. Im Folgenden seien die rechtlichen, insbesondere aufklärungsrechtlichen Bedingungen solcher Wahlentscheidungen erörtert.

Die umfassende Transparenz und gute Kommunikation über die Impfstoffe ist die Voraussetzung einer breiten Annahme der Impfung in der Bevölkerung ${ }^{2}$. Dies erfordert eine sachgemäße und sachliche Information über die Impfstoffe, ihren Nutzen und die unerwünschten Wirkungen und impliziert, dass dabei auch über die Begrenztheit unseres bisherigen Wissens informiert wird. Die individuelle Aufklärung der zu Impfenden ist ein wichtiger Prozess und Teil der notwendigen allgemeinen Information und Beratung über die Impfstoffe ${ }^{3}$. Aufklärung ist ein wichtiger Teil der Legitimation der Impfung und ihrer Praxis. Gute Aufklärung ist gute Kommunikation und gegebenenfalls „kommunikative Partizipation“"4.

Der Umfang und der Inhalt der Aufklärung über den zu applizierenden Impfstoff sind abhängig von unserem Wissen über seine Eigenschaften. Impfstoffe sind Arzneimittel. Die Aufklärung der zu Impfenden erfolgt auf behandlungsvertraglicher Basis und richtet sich insofern nach den Grundsätzen der Aufklärung bei der Arzneimittelbehandlung (\$S 630c Abs. 2, 630e Abs. 1 BGB). Die Aufklärung hat individuell, situationsbezogen, mündlich und zeitgerecht durch einen Arzt zu erfolgen ${ }^{5}$. Sie umfasst auch die Aufklärung über Behandlungsalternativen, wenn ,gleichermaßen indizierte und übliche Methoden zu wesentlich unterschiedlichen Belastungen, Risiken oder Heilungschancen führen können. "(\$630e Abs. 1 S. 3 BGB).

$\mathrm{Ob}$ die Situation konkurrierender Impfstoffe ohne oder mit unterschiedlichen Eigenschaften aufklärungsrechtlich und gegebenenfalls wie zu behandeln ist, stellt aufklärungsrechtlich die Frage, ob die Wahl zwischen zur Verfügung stehenden unterschiedlichen Arzneimitteln/Impfstoffen identischer Indikation in die ärztliche Methodenfreiheit fällt und deshalb der ärztlichen Entscheidung unterliegt oder ob diese Wahl der Selbstbestimmung der Patienten überlassen bleibt. Eine andere Situation besteht im Falle der Impfstoffknappheit - dort regiert Knappheit die Anwendung.

Die erste Problematik wird in Literatur und Rechtsprechung unter dem Stichwort der Alternativenaufklärung abgehandelt. Zwei Typen solcher Konkurrenz sind zu unterscheiden: Es stehen sich zwei oder mehrere Arzneimittel mit vergleichbarem Wirksamkeits- und Risikoprofil [= vergleichbare Nutzen] (6) oder zwei oder mehrere Arzneimittel mit unterschiedlichen Wirksamkeits- und Risikoprofilen bzw. deren Verhältnis [= unterschiedliche Nutzen] (7) gegenüber. Zuvor ist zu klären, welche Nutzenbewertungen

Prof. Dr. iur. Dieter Hart,

Institut für Informations-, Gesundheits- und Medizinrecht, Universität Bremen,

Postfach 330 440, 28334 Bremen, Deutschland für die Qualifikation der Impfstoffe als vergleichbar oder unterschiedlich im Infektionsschutz-, Gesetzlichen Krankenversicherungsrecht (GKV-Recht) und im Behandlungsvertragsrecht ausschlaggebend sind: die Urteile der Zulassungsbehörden (EMA, PEI) oder/und zusätzlich oder alleine der mit Empfehlungen beauftragten unabhängigen Stellen (z.B. STIKO) (2). Die Typen der Impfstoffe (3), die zugelassenen bzw. kurz vor der Zulassung stehenden Impfstoffe und ihre wesentlichen Eigenschaften werden im Vergleich charakterisiert (4) und das infektions- und sozialrechtliche Verhältnis von Knappheit, Priorisierung und Aufklärung (5) wird kurz präsentiert. Die Einordnung der verschiedenen Impfstoffe in die Aufklärungssystematik erfolgt am Ende (8).

\section{Institutionen der Nutzenbewertung}

Impfstoffe gelten gem. $₫ 4$ Abs. 4 i.V.m. $₫ 2$ Abs. 1 AMG als Arzneimittel. Zuständig für die Nutzenbewertung von Arzneimitteln und Impfstoffen sind auf EU-Ebene die EMA (Europäischen Arzneimittel-Agentur), auf nationaler Ebene das BfArM (Bundesinstitut für Arzneimittel und Medizinprodukte) für Arzneimittel ( $\$ 77$ Abs. 1 AMG) und das PEI (Paul Ehrlich Institut) für Impfstoffe (\$77 Abs. 2 AMG). Darüber hinaus sind an regulatorischen Arzneimittelbewertungen sozialrechtlich das IQWiG und der G-BA und als unabhängige Empfehlungskommission beim RKI (Robert Koch Institut) die STIKO für Impfstoffe (Ständige Impfkommission) sowie auf fachlicher Ebene medizinische Fachgesellschaften und privatwissenschaftliche, auch unabhängige publizistische Institutionen (z.B. ARZNEIMITTELBRIEF, arznei-telegramm) beteiligt.

Eine Bewertungshierarchie oder ein Bewertungszusammenspiel der genannten Institutionen existiert je nach Rechtsgebiet und Regelungstyp. Es ist zu klären, ob und gegebenenfalls welche Konfliktkonstellationen entstehen können.

1) https://www.spiegel.de/wissenschaft/corona-news-am-8-2-158tote-und-mehr-als-4500-corona-neuinfektionen-in-deutschlanda-b181263d-fab2-4213-acdd-add2c0abe6fc (abgerufen 8.2.2021).

2) Dazu Drechsler, Selbstbestimmungsaufklärung und Pandemiebekämpfung, MedR 2021 (erscheint demnächst).

3) $\mathrm{Zu}$ den Anforderungen an die Aufklärung über die Impfstoffe Thesenpapier 7: Schrappe/François-Kettner/Gruhl/Hart/Knieps/Manow/Pfaff/Püschel/Glaeske, Die Pandemie durch SARS-CoV-2/ COVID-19 (Thesenpapier 7): Sorgfältige Integration der Impfung in eine umfassende Präventionsstrategie, Impfkampagne resilient gestalten und wissenschaftlich begleiten, Aufklärung und Selbstbestimmung beachten., Köln, Berlin, Bremen, Hamburg, 10.1.2021, Monitor Versorgungsforschung online, http://doi.org/ 10.24945/MVF.1.21.1866-0533.2268, Abschnitt 3.3.4.

4) Zu Letzterem Damm, in: Katzenmeier (Hrsg.), FS f. Dieter HartMedizin - Recht - Wissenschaft, 2020, S. 81 ff., 98 ff. Es sei darauf hingewiesen, dass die Fälle von $\$ 630$ e Abs. 3-5 BGB hier nicht behandelt werden.

5) Dazu allgemein Katzenmeier, in: Laufs/Katzenmeier/Lipp, Arztrecht, 8. Aufl. 2021, Kap. V, S. 113 ff.; speziell Hart, Arzneimittelbehandlung, in: Rieger/Dahm/Katzenmeier/Stellpflug/Ziegler (Hrsg.), HKAKM, Stichwort Nr. 240, Rdnrn. 21 ff.; ders., Aufklärung bei der Arzneimittelbehandlung, ebd., Stichwort Nr. 643, Rdnrn. $20 \mathrm{ff}$. 


\subsection{GKV-Recht}

Die EU- oder nationale Zulassung von Arzneimitteln sind für das GKV-Recht nach der ständigen Rechtsprechung des BSG vorgreiflich ${ }^{6}$. Jedes Arzneimittel, das über eine solche Zulassung verfügt, kann in der GKV verschrieben werden, sofern es nicht durch Gesetz, Rechtsverordnung oder die Arzneimittel-Richtlinie des G-BA aus dem Leistungskatalog ausgeschlossen ist; es ist GKV-fähig. Dasselbe gilt für Impfstoffe.

Die bisher in der EU zugelassenen Impfstoffe sind zwar GKV-fähig, aber sie unterliegen dem besonderen Regime der Entscheidungsermächtigung des BMG nach SGB V und IfSG. Nach $₫ 5$ Abs. 2 S. 1 Nr. 4 a IfSG ist der BMG ermächtigt

„durch Rechtsverordnung ohne Zustimmung des Bundesrates Maßnahmen zur Sicherstellung der Versorgung mit Arzneimitteln einschließlich Impfstoffen und Betäubungsmitteln .... zu treffen und insbesondere

a) Ausnahmen von den Vorschriften des Arzneimittelgesetzes, des Betäubungsmittelgesetzes, des Apothekengesetzes, des Fünften Buches Sozialgesetzbuch, des Transfusionsgesetzes sowie der auf ihrer Grundlage erlassenen Rechtsverordnungen, ..., zuzulassen ...

c) Maßnahmen zum Bezug, zur Beschaffung, Bevorratung, Verteilung und Abgabe solcher Produkte durch den Bund $\mathrm{zu}$ treffen sowie Regelungen zu Melde- und Anzeigepflichten vorzusehen, ...

f) Regelungen zum Vertrieb, zur Abgabe, Preisbildung und -gestaltung, Erstattung sowie Vergütung vorzusehen, ..."

und zusätzlich nach $\$ 20$ i Abs. 3 S. 2 Nr. 1 Buchstabe a und Nr. 2, S. 3, 7 und 8 sowie 10 bis 11 SGB V eine Impfverordnung zu erlassen. Die Verordnung zum Anspruch auf Schutzimpfung gegen das Coronavirus SARS-CoV-2 vom 8.2.2021 (Coronavirus-Impfverordnung - Corona-ImpfV) ${ }^{7}$ ist auf dieser Basis erlassen worden und regelt die Leistungsansprüche der Versicherten, die Kostentragung und anderes $^{8}$. Die STIKO ist bei Entscheidungen über die Impfstoffe gegen Covid-19 nach $\$ 20$ i Abs. 3 S. 8 SGB V anzuhören; der G-BA hat nach $\$ 20$ i Abs. 3 S. 14 SGB V unter besonderen Voraussetzungen die Einzelheiten zu Voraussetzungen, Art und Umfang von Schutzimpfungen zu bestimmen. Die Empfehlungen der STIKO bezüglich Alter und Zeitabständen der Folgeimpfungen werden durch die $\$ \$ 2$ Abs. 2 S. 1, 3 Abs. 2, 4 Abs. 2 und 5 CoronaImpfV verpflichtend'.

Die CoronaImpfV v. 8.2.2021 ist durch die CoronaImpfV v. 10.3.2021 mit Wirkung zum 8.3.2021 abgelöst worden ${ }^{10}$. Die Neufassung enthält keine Altersbegrenzungen für den AstraZenenca-Impfstoff mehr, so dass die Regelungen in den früheren $\int \$ 2$ Abs. 2 S. 1, 3 Abs. 2, 4 Abs. 2 und 5 CoronaImpfV alt entfallen.

Nach der EU-Zulassung auf der Basis der EMA-Empfehlung ist der Impfstoff verkehrsfähig und nach der weiteren Empfehlung der STIKO ist der Impfstoff im Rahmen der GKV als der erforderlichen Qualität und Wirksamkeit nach dem allgemein anerkannten Stand der medizinischen Erkenntnisse entsprechend ( $\$ 2$ Abs. 1 S. 3 SGB V) einsetzbar. $\mathrm{Zu}$ den Anforderungen des GKV-Rechts an die Aufklärung sei auf die jüngste Entscheidung des BSG und deren Besprechungen verwiesen ${ }^{11}$.

Nach $₫ 1$ Abs. 1 CoronaImpfV haben alle in Deutschland gesetzlich oder privat versicherten und Personen, die ihren Wohnsitz oder gewöhnlichen Aufenthaltsort in der Bundesrepublik Deutschland haben sowie weitere dort aufgezählten Personen ,im Rahmen der Verfügbarkeit“ einen Anspruch auf die Impfung, bei Impfstoff knappheit nach der in $\int 1$ Abs. 2 CoronaImpfV geregelten Reihenfolge der Priorisierung.
Der Anspruch ist also begrenzt: er besteht nur ,im Rahmen der Verfügbarkeit der vorhandenen Impfstoffe". Dieser Rahmen nimmt in erster Linie Bezug auf die Priorisierungen als erforderlichen Konsequenzen aus dem (anfänglichen) Mangel an Impfstoff. In der Begründung zum Referentenentwurf der CoronaImpfV findet sich zu $\$ 1$ der Satz ${ }^{12}$ :

„Darüber hinaus beinhaltet der Anspruch auf Schutzimpfung gegen das Coronavirus SARSCoV-2 nicht das Recht, den Impfstoff eines bestimmten Herstellers zu wählen.“

Eine wörtliche Auslegung des $₫ 1$ Abs. 1 CoronaImpfV deckt eine solche Auslegung jedenfalls nicht allgemein. Auch systematisch und teleologisch ist eine solche allgemeine Begrenzung des Anspruchs nicht gedeckt. Sofern der Mangel an Impfstoffen nicht (mehr) besteht, ist der Grund für eine Versagung eines Auswahlrechts nicht mehr vorhanden. Im Falle, dass unterschiedliche Arzneimittel identischer Indikation zugelassen und verfügbar sind sowie dem allgemein anerkannten Stand der medizinischen Erkenntnisse entsprechen, ist der Leistungsanspruch des GKV-Versicherten nach $\int S 27$ Abs. 1 S. 2 Nr. 3, 31 SGB V - außer im Falle von Ausschlüssen ${ }^{13}$ - nicht begrenzt ${ }^{14}$, sondern die Wahl des Arzneimittels obliegt im Rahmen der ärztlichen Verordnung und des Behandlungsverhältnisses ${ }^{15}$ dem jeweiligen Patienten ${ }^{16}$. Dasselbe gilt für Impfstoffe, es sei denn, die Wahlfreiheit sei gesetzlich begrenzt. Die hier vertretende Auslegung von $\$ 1$ Abs. 1 CoronaImpfV begründet jedenfalls keinen allgemeinen Ausschluss des

6) Überblick über Literatur und Rechtsprechung bei Hart, Arzneimittelzulassung, in: Rieger/Dahm/Katzenmeier/Stellpflug/Ziegler, HK-AKM, Nr. 320, Stand Juli 2018, Rdnrn. 28f; ders., off-labeluse, ebd., Nr. 3910, Stand April 2013, Rdnrn. 91 ff., $111 \mathrm{ff}$.

7) CoronaImpfV v. 8.2.2021, BAnz. AT 8.2.2021 V1.

8) Die im Entwurf eines Gesetzes zur Fortgeltung der die epidemische Lage von nationaler Tragweite betreffenden Regelungen, BT-Dr. 19/26545 v. 9.2.2021 vorgesehenen Änderungen des IfSG und des SGB V ändern diese Regelungen nicht.

9) Zur Frage der ausreichenden Rechtsgrundlage für die in der CoronaImpfV vorgesehenen Priorisierungen s. Kießling/Kingreen/ Leisner-Egensperger, Keine Priorität für die Rechtswissenschaft: Als Rechtsgrundlage einer Impfpriorisierung dürfte die CoronaImpfverordnung vor den Verwaltungsgerichten schwerlich Bestand haben, VerfBlog, 2021/2/02, https://verfassungsblog.de/keineprioritat-fur-die-rechtswissenschaft/, DOI: 10.17176/20210202235755-0; Rixen, Impfschutzrecht, in: Huster/Kingreen (Hrsg.), Handbuch Infektionsschutzrecht, 2021, Kap. 5, Rdnrn.13ff.

10) CoronaImpfV v. 10.3.2021, BAnz. AT 11.3.2021 V1.

11) BSG, Urt. v. 19.3.2020 - B 1 KR 20/19 R-, MedR 2020, 941; dazu Felix, MedR 2021, $7 \mathrm{ff}$; Hart, MedR 2020, $895 \mathrm{ff}$

12) CoronaImpfV, RefE BMG, Begründung zu $\$ 1$, S. 22, https:// www.bundesgesundheitsministerium.de/fileadmin/Dateien/3_Downloads/C/Coronavirus/Verordnungen/CoronaImpfV_mit_Begruendung_080221.pdf (zuletzt abgerufen am 12.2.2021); identisch im RefE der neuen CoronaImpfV Stand 9.3.2021, S. 24, https://www.bundesgesundheitsministerium.de/ fileadmin/Dateien/3_Downloads/C/Coronavirus/Verordnungen/CoronaImpfV_RefE_mit_Begruendung_100321.pdf.

13) Kraft Gesetzes (z.B. $\$ 34$ SGB V), durch Rechtsverordnung oder durch untergesetzliche Regelung: Arzneimittel-Richtlinie des G-BA gemäß $\$ 92$ Abs. 1 S. 2 Nr. 6 SGB V, i.d.F. v. 18.12.2008/22.1.2009, veröffentlicht im BAnz. Nr. 49a (Beilage) v. 31.3.2009, zuletzt geändert am 17.12.2020, veröffentlicht im BAnz. (BAnz. AT 18.1.2021 B7), in Kraft getreten am 19.1.2021, \$S $12 \mathrm{ff}$.

14) Dazu allgemein Becker/Kingreen/Axer, 7. Aufl. 2020, SGB V, $₫ 31$, Rdnrn. 20-25.

15) Klarstellung durch $₫ 630$ a Abs. 1 BGB, $\$ 76$ Abs. 4 SGB V bestätigend, Spickhoff/Nebendahl, 3. Aufl. 2018, SGB V \$76, Rdnrn. 15-17; Becker/Kingreen/Lang, SGB V \$76, Rdnrn. 25, 26; zu den Pflichten aus dem Behandlungsverhältnis gleich anschließend.

16) Vgl. Pitz, in: Schlegel/Voelzke, jurisPK-SGB V, 4. Aufl., $₫ 31$ SGB V (Stand: 5.11.2020), Rdnrn. $117 \mathrm{ff}$ 
Wahlrechts. Sofern also eine Mangelsituation nicht mehr gegeben ist, bleibt es beim Wahlrecht. D.h.: spätestens, wenn die Priorisierungsgruppen bedient sind, entsteht ein Wahlrecht. Der Mangel an Impfstoff kann allerdings auch schon vor dieser Situation aufgehoben sein, wenn nämlich unterschiedliche Impfstoffe mangelfrei für alle Impfberechtigten angeboten werden können und die Priorisierung ihren Grund ausschließlich in der Bevorzugung der Risikogruppen, nicht aber im Mangel hat. Der Mangel entfällt aber auch schon dann risikogruppenspezifisch, wenn die erste, zweite, dritte Priorisierungsgruppe ,durchgeimpft“" ist.

Der Anspruch umfasst nach $\$ 1$ Abs. 4 CoronaImpfV die folgenden Leistungen:

„(3) Der Anspruch nach Abs. 1 S. 1 umfasst die Aufklärung und Impfberatung der zu impfenden Person, die symptombezogene Untersuchung zum Ausschluss akuter Erkrankungen oder Allergien, die Verabreichung des Impfstoffes, die Beobachtung der sich an die Verabreichung des Impfstoffes unmittelbar anschließenden Nachsorgephase und erforderliche medizinische Intervention im Falle des Auftretens von Impfreaktionen. ... .“

Auch die Leistung Aufklärung wird dort in $\ 1$ Abs. 4 S. 2 CoronaImpfV umrissen.

„Die Aufklärung und Impfberatung der zu impfenden Person beinhalten:

1. die Information über den Nutzen der Schutzimpfung und die Coronavirus-Krankheit-2019 (COVID-19),

2. die Erhebung der Anamnese einschließlich der Impfanamnese sowie der Befragung über das Vorliegen möglicher Kontraindikationen,

3. die Feststellung der aktuellen Befindlichkeit zum Ausschluss akuter Erkrankungen oder Allergien,

4. Hinweise auf mögliche Nebenwirkungen und Komplikationen der Schutzimpfung,

5. die Informationen über den Eintritt und die Dauer der Schutzwirkung der Schutzimpfung,

6. Hinweise zu Folge- und Auffrischimpfungen,

7. Empfehlungen über Verhaltensmaßnahmen im Anschluss an die Schutzimpfung."

Die Umsetzung des Anspruchs erfolgt in einem behandlungsvertragsrechtlichen Rahmen (\$630a Abs. 1 BGB).

\subsection{Vertrags- und Haftungsrecht}

Die Nutzenbewertung ist im Haftungsrecht auf der Ebene der Behandlung (2.2.1) wie auf der der Aufklärung (2.2.2) relevant.

\subsubsection{Behandlung}

Im Vertragsrecht gilt gemäß $\$ 630$ a Abs. 2 BGB gilt , der allgemein anerkannte fachliche Standard“. Der die Haftung auslösende Fehler ist die Unterschreitung dieses Standards ${ }^{17}$. Bei den Impfstoffen kommt es zunächst auf die Impfstoffeigenschaften nach der Fach- bzw. Gebrauchsinformation und darüber hinaus auf die durch neuere Studien zur Anwendung der Impfstoffe, durch die Sicherheitsberichte der EMA ${ }^{18}$ und des $P E I^{19}$ oder durch ärztliche Erfahrung sich aktualisierenden Wirkungen und Eigenschaften der Impfstoffe an. Von besonderer Bedeutung bei der Impfung gegen Covid-19 sind die Behandlungsstationen (und in der Folge Fehlertypen bei) der Anamnese, der Indikation und der Therapie:

1. Im Rahmen der Anamnese sind insbesondere der gegenwärtige Gesundheitsstatus und Vorerkrankungen zu erheben, wobei Letztere den Einsatz eines jeden oder nur eines einzelnen Impfstoffs ausschließen können.
Ausschlaggebend sind in erster Linie die Zulassung, die darauf beruhende Fachinformation und alle Pharmakovigilanzdaten aus Nachzulassungsstudien und wissenschaftlich aufbereiteten Spontanmeldungen.

2. Im Rahmen der Indikation geht es insbesondere um z.B. die Altersabhängigkeit der Anwendung (Kinder, Jugendliche, Ältere). Ausschlaggebend sind in erster Linie die Zulassung und die darauf beruhenden Fachinformation, aber auch insofern können Nachzulassungsdaten relevant werden.

3. Im Rahmen der Therapie geht es um eine ,therapeutische Nutzen- und Gefahrenvorsorge" bei der Auswahl des Impfstoffs ${ }^{20}$. Die vergleichende Bewertung des Nutzens der Impfstoffe kann bei relevanten Eigenschaftsunterschieden eine Auswahl zugunsten des einen oder anderen erfordern. Ausschlaggebend sind neben der Zulassung, der darauf beruhenden Fachinformation neuere Erkenntnisse aufgrund Nachzulassungsstudien und Sicherheitsberichten.

Abweichungen zwischen GKV-Recht, Vertrags- und Haftungsrecht existieren insofern nicht.

\subsubsection{Aufklärung}

Sowohl für die Sicherungs- wie die Selbstbestimmungsaufklärung sind die einleitend genannten Bewertungsinstitutionen grundlegend. Mit der Entwicklung der Kenntnisse über die Impfstoffe entwickeln sich auch die Aufklärungserfordernisse. Die kontinuierliche Aktualisierung der Datenlage über Wirkungen und unerwünschte Wirkungen der Impfstoffe erfordert entsprechende Anpassungen des Aufklärungsprogramms und die Übernahme neuer Informationen in die Aufklärung, soweit dadurch die selbstbestimmte Entscheidung der zu Impfenden beeinflusst werden kann. Dasselbe gilt für die Ergebnisse der Pharmakovigilanz und der sonstigen geplanten Studien ${ }^{21}$. Insofern sind alle Nachzulassungsinformationen für die Aufklärung ausschlaggebend.

Die Verpflichtungen zur Aufklärung gelten gleichermaßen für Patienten wie Versicherte. Abweichungen zwischen GKVRecht, Vertrags- und Haftungsrecht existieren insofern nicht.

\section{Profile der Impfstofftypen}

Unterschieden werden mRNA- (BioNTech, Moderna, CureVac), und virale Vektor-Impfstoffe (AstraZeneca,

17) Insgesamt dazu Jansen, Der medizinische Standard - Begriff und Bestimmung ärztlicher Behandlungsstandards an der Schnittstelle von Medizin, Haftungsrecht und Sozialrecht, Kölner Schriften zum Medizinrecht Bd. 25, Berlin 2019, bes. S. $38 \mathrm{ff.,} 183 \mathrm{ff}$.; Hart, in: Rieger/Dahm/Katzenmeier/Stellpflug/Ziegler, HK-AKM, Nr. 240, Stand Februar 2016, Rdnrn. 9 ff.

18) Überblick https://www.ema.europa.eu/en/news/ema-publishessafety-monitoring-plan-guidance-risk-management-planningcovid-19-vaccines und Pharmakovigilanzplan https://www. ema.europa.eu/en/documents/other/pharmacovigilance-planeu-regulatory-network-covid-19-vaccines_en.pdf (zuletzt abgerufen 8.2.2021).

19) S. https://www.pei.de/DE/newsroom/dossier/coronavirus/arzneimittelsicherheit.html (zuletzt abgerufen 8.2.2021).

20) Hart, in: Rieger/Dahm/Katzenmeier/Stellpflug/Ziegler, HK-AKM, Nr. 240, Stand Februar 2016, Rdnrn. $15 \mathrm{ff}$.

21) Vgl. STIKO-Empfehlung zur COVID-19-Impfung, Epidemiologisches Bulletin 2 | 2021 14.1.2021 (online vorab) mit Aktualisierung v. 8.1.2021, Kap. 16.2, https://www.rki.de/DE/ Content/Infekt/EpidBull/Archiv/2021/Ausgaben/02_21.pdf? _blob=publicationFile und 2. Aktualisierung vom 4.2.2021 https://www.rki.de/DE/Content/Infekt/EpidBull/Archiv/ 2021/Ausgaben/05_21.pdf?_blob=publicationFile (zuletzt abgerufen 8.2.2021); dazu die Sicherheitsberichte des PEI, zuletzt Bericht v. 4.3.2021 bis zum 26.2.2021, https://www.pei.de/DE/ newsroom/dossier/coronavirus/coronavirus-inhalt.html?cms pos $=5$ (zuletzt abgerufen am 5.3.2021). 
Johnson\&Johnson, Sputnik V, Sinovac). Zusätzlich sind Protein-Impfstoffe in der Prüfung (Novavax).

Ein mRNA-basierter Impfstoff (mRNA = Messenger-Ribonukleinsäure) enthält den „Bauplan“ (Abschrift eines Gens) für ein Eiweiß auf der Oberfläche des Coronavirus SARS-CoV-2. Durch die Impfung gelangt dieser Bauplan mit Hilfe von Lipid-Nanopartikeln, die die mRNA „,verpacken“, leichter in die Körperzellen. Sie stellen dann für eine kurze Zeit das Spike-Glykoprotein her, dann wird die mRNA in den Zellen abgebaut. Dadurch wird das Immunsystem angeregt, Abwehrstoffe (neutralisierende Antikörper und T-Zellen) gegen das Spike-Glykoprotein zu bilden. Wenn die geimpfte Person später in Kontakt mit SARS-CoV-2 kommt, wird das Spike-Glykoprotein des Virus schnell durch das Immunsystem erkannt und gezielt bekämpft.

Der Vektor-Impfstoff besteht aus den „Hüllen“ harmloser (Adeno-)Viren, die ebenfalls den „Bauplan“ (Abschrift der DNA) für das Spike-Glykoprotein auf der Oberfläche des Coronavius SARS-CoV-2 enthalten. Diese Vektoren werden von Körperzellen aufgenommen, die dann für eine kurze Zeit dieses Corona-Eiweiß (S-Protein) herstellen. Dadurch wird das Immunsystem angeregt, Abwehrstoffe (Antikörper und T-Zellen) gegen das S-Protein zu bilden. Wenn die geimpfte Person später in Kontakt mit SARSCoV-2 kommt, wird dieses Virus schnell durch das Immunsystem erkannt und gezielt bekämpft ${ }^{22}$.

Vektor-Impfstoffe basieren auf umfangreicheren Erfahrungen, während die mRNA-Impfstoffe eine in anderen Zusammenhängen zwar getestete, aber im Prinzip neue Entwicklungsform darstellen. Die mRNA-Impfstoffe scheinen für Mutationen des Virus, die natürlicherweise und regelmäßig vorkommen, leichter anpassbar zu sein als die Vektor-Impfstoffe, bei denen möglicherweise zusätzlich (starke) Immunreaktionen gegen das Adenovirus zu berücksichtigen sind. Vektor-Impfstoffe sind unkomplizierter in der Lagerung und Anwendung und leichter zu transportieren. Diese allgemeinen Unterschiede erschweren die Vergleichbarkeit der Impfstoffe, zumal keine vergleichenden klinischen Studien zu mRNA- und Vektor-Impfstoffen vorliegen. Diese Erschwernis ist bei den indikations- und aufklärungsrechtlichen rechtlichen Nutzen-Vergleichen zu berücksichtigen.

In der Entwicklung befinden sich zusätzlich noch Protein-Impfstoffe ${ }^{23}$, für die die EU bisher nur einen Liefervertrag geschlossen hat. Daten aus Phase III-Prüfungen liegen bisher nicht vor, so dass sie hier auch nicht berücksichtigt werden.

\section{Die Impfstoffe ${ }^{24}$ und ihre wesentlichen Eigenschaften}

Verf. bezieht sich insbesondere auf EMA-Informationen ${ }^{25}$ (Packungsbeilagen), die (auch verlinkten) Informationen des PEI ${ }^{26}$, Veröffentlichungen der Fachliteratur, von Lancet bis ARZNEIMITTELBRIEF ${ }^{27}$, und entnimmt ihnen wesentliche Charakterisierungen der Impfstoffe, die insbesondere auf Studien der Phase III $^{28}$ bzw. Interimsanalysen solcher Studien beruhen ${ }^{29}$. Darüber hinaus werden neuere Informationen aus Studien oder Interimsstudien und aus Sicherheitsberichten von EU- oder nationalen Behörden genutzt ${ }^{30}$. Die folgenden Angaben sind auf einem Stand von Ende Februar (teilweise Anfang März) 2021; sie können sich nach weiteren, den pharmazeutischen Unternehmern aufgegebenen Studien und den Pharmakovigilanzdaten verändern und wären demgemäß anzupassen. Insgesamt beziehen sich die relevanten Kenntnisse über die Eigenschaften der Impfstoffe auf Interimsanalysen von Phase III-Studien. Verf. versucht gewisse Nutzentypisierungen vorzunehmen, die natürlich vorläufig sind. Von großer Bedeutung wird die meldungsbedingte und systematische Pharmakovigilanz sein, weil die bisherigen Kenntnisse sich nur auf akut bzw. früh auftretende unerwünschte Impfstoffeigenschaften beziehen. Insofern sind die Sicherheitsberichte der EMA und des PEI ${ }^{31}$ über die Impfstoffanwendung von erheblicher Relevanz auch für die Aufklärung der zu Impfenden und ihre Einordnung in die Alternativenaufklärung.

Alle Bewertungen sind unter den Anforderungen einer Evidenz-basierten Medizin nur mit Vorsicht $\mathrm{zu}$ behandeln und es sei nochmals betont: Es handelt sich um Bewertungen unter erheblicher Unsicherheit, da (häufig) im Gegensatz zu Arzneimitteln keine vergleichenden Prüfungen zwischen den Impfstoffen vorliegen. Auch die Qualität der Studien schwankt. Insofern handelt es sich im Folgenden um Annäherungen an Vergleiche unter Unsicherheit ${ }^{32}$.

Die ersten drei Impfstoffe sind mRNA-Impfstoffe, die folgenden zwei Vektorimpfstoffe. Novavax ist ein ProteinImpfstoff. Über diese sechs Impfstoffe hat die EU Lieferverträge abgeschlossen.

22) Beide Beschreibungen zitiert aus IQWiG Gesundheitsinformation, https://www.gesundheitsinformation.de/der-impfstoff-comirnaty-bnt162b2-biontech-pfizer-zur-impfung-gegen-corona html und https://www.gesundheitsinformation.de/der-impfstoff-astrazeneca-azd1222-astrazeneca-zur-impfung-gegen-corona.html (zuletzt abgerufen 6.2.2021).

23) S. Überblick über die in der Entwicklung befindlichen und zugelassenen Impfstoffe unter https://vac-lshtm.shinyapps.io/ ncov_vaccine_landscape/. Beispiel für einen Protein-Impfstoff: Fougeroux et al., Capsid-like particles decorated with the SARSCov-2 receptor-binding domain elicit strong virus neutralization acivity, Nature Communications 12, Article Number 324 (2021), https://www.nature.com/articles/s41467-020-20251-8.

24) Die Auswahl berücksichtigt Impfstoffe, die in der EU bedingt zugelassen sind, einen Zulassungsantrag gestellt haben oder ein Zulassungsverfahren erwarten (Firmen, mit denen die EU Lieferverträge abgeschlossen hat). Sanofi GSK wird wegen der noch unklaren Entwicklungszeit und fehlender Daten nicht berücksichtigt. Inzwischen ist auch Sputnik V bei der EMA im RollingReview-Verfahren.

25) Z.B. BioNTech/Pfizer Comirnaty, https://www.ema.europa. $\mathrm{eu} / \mathrm{en} /$ documents/product-information/comirnaty-epar-product-information_de.pdf (zuletzt abgerufen 17.2.2021).

26) Überblick über die zugelassenen Impfstoffe https://www.pei.de/ DE/arzneimittel/impfstoffe/covid-19/covid-19-node.html;jsessionid $=0 \mathrm{DB} 7402 \mathrm{C} 4545267 \mathrm{~B} 2 \mathrm{D} 1 \mathrm{~F} 8102658 \mathrm{D} 6858$. intranet 221 (zuletzt abgerufen 17.2.2021).

27) Die beiden ersten zugelassenen mRNA-Impfstoffe gegen COVID-19, AMB 2021,55, 01 ff.; zum AstraZeneca-Impfstoff AMB 2/2021, 55, 13 ff.; s. auch die Informationen des IQWiG, z.B. https://www.gesundheitsinformation.de/der-impfstoff-comirnaty-bnt162b2-biontech-pfizer-zur-impfung-gegen-corona. html, dort auch Informationen zu Moderna und AstraZeneca (zuletzt abgerufen 6.2.2021); zu AstraZeneca arznei-telegramm 2021, 52, 2/9ff.

28) Hart, Klinische Arzneimittelprüfung, in: Rieger/Dahm/Katzenmeier/Stellpflug/Ziegler, HK-AKM, Nr. 2880, Stand Juli 2016, Rdnrn. $1 \mathrm{ff}$., $14 \mathrm{ff}$., $21 \mathrm{ff}$.

29) Z.B. Denis Y Logunov u. a., Safety and efficacy of an rAd26 and rAd5 vector-based heterologous prime-boost COVID-19 vaccine: an interim analysis of a randomised controlled phase 3 trial in Russia, published: February 02, 2021, DOI:https://doi.org/10. 1016/S0140-6736(21)00234-8.

30) S. auch allgemein zu den Impfstoffen, ihrer Produktion und ihren Preisen weltweit Oliver J Wouters u. a., Challenges in ensuring global access to Covid-19 vaccines: production, affordability, allocation and development, thelancet.com, published online February 12, 2021 https://doi.org/10.1016/S0140-6736(21)00306-8 (zuletzt abgerufen 17.2.2021).

31) Zuletzt Sicherheitsbericht v. 4.3.2021 bis zum 26.2.2021, https://www.pei.de/SharedDocs/Downloads/DE/newsroom/ dossiers/sicherheitsberichte/sicherheitsbericht-27-12-bis-26$02-21$.pdf?__blob $=$ publicationFile $\& \mathrm{v}=8$ (zuletzt abgerufen am 5.3.2021).

32) Zur schwierigen Vergleichbarkeit der Impfstoffe auch AMB 2/2021, 55, 13, 15 . 
1. BioNTech/Pfizer ${ }^{33}$ (mRNA-Impfstoff; BNT162b2; Comirnaty $^{\circledR}$; bedingt zugelassen in EU) Wirksamkeit

$\left(\right.$ relativ $\left.^{34}\right) 95 \%$; verhindert über einen Zeitraum von mindestens 2-3 Monaten mindestens symptomatische Infektionen und schwere Krankheitsverläufe; unklar ob sterile Immunität $t^{35}$ erreicht wird; Wirksamkeitsaussage für Personen ab 70 Jahren beruhen auf recht geringen Zahlen. Wirksam gegen britische (B.1.1.7), weniger wirksam gegen südafrikanische (B.1.351) Mutation; schützt möglicherweise vor Infektion.

\section{Nebenwirkungen}

etwa $75 \%$ der Geimpften haben leichte bis mäßige lokale, $50 \%$ systemische unerwünschte Arzneimittelereignisse (UAW); häufiger nach der 2. Impfung und häufiger bei Jüngeren als Älteren.

Gegenanzeigen

Immunsupprimierte Personen, Schwangere und Jugendliche sind bisher nicht untersucht; von Studien ebenfalls ausgeschlossen Personen mit Anaphylaxie; deshalb keine Impfung; Warnhinweis der EMA bei schweren allergischen Reaktionen.

Es handelt sich um eine bedingte Zulassung der EU, bei der kein Unterschied bezüglich der Altersgruppen gemacht wurde; zugelassen ab 16 Jahre.

2. Moderna ${ }^{36}$ (mRNA-Impfstoff; COVID-19 Vaccine Moderna; mRNA-1273; bedingt zugelassen in EU) Wirksamkeit

(relativ) 94,1\%; verhindert über einen Zeitraum von mindestens 2-3 Monaten mindestens symptomatische Infektionen und schwere Krankheitsverläufe; unklar ist, ob sterile Immunität erreicht wird; Wirksamkeitsaussagen für Personen ab 70 Jahren beruhen auf recht geringen Zahlen; Schutz vor Mutationen noch unklar.

Nebenwirkungen

etwa $80 \%$ der Geimpften haben leichte bis mäßige lokale, etwa $60 \%$ systemische unerwünschte Arzneimitteereignisse (UAW); häufiger nach der 2. Impfung und häufiger bei Jüngeren als Älteren.

Gegenanzeigen

Immunsupprimierte Personen, Schwangere und Jugendliche sind bisher nicht untersucht; deshalb keine Impfung; Warnhinweis der EMA bei schweren allergischen Reaktionen.

Es handelt sich um eine bedingte Zulassung der EU, bei der kein Unterschied bezüglich der Altersgruppen gemacht wurde; zugelassen ab 18 Jahre.

3. CureVac (mRNA Impfstoff; noch kein Zulassungsnatrag gestellt, aber Rolling-Review-Verfahren bei EMA eingeleitet ${ }^{37}$; Liefervertrag mit EU abgeschlossen; bisher keine Daten aus Phase III-Studien)

4. AstraZeneca ${ }^{38}$ (Vector-Impfstoff; bedingt zugelassen in EU)

Wirksamkeit

(relativ) etwa $70 \%$; geringer Schutz nach erster, voller Schutz nach zweiter Dosis, jeweils nach 2 Wochen; verhindert möglicherweise zu $100 \%$ Krankenhausbehandlungen und schwere Krankheitsverläufe; Wirksamkeitsaussage für Personen ab 70 Jahren beruhen auf recht geringen Zahlen (6\%); wahrscheinlich wirksamer Schutz gegen britische, möglicherweise kein oder geringerer Schutz vor südafrikanischer Mutation.

Die Aussage zur Wirksamkeit bei halber Erstdosis und zweiter Volldosis (also entgegen der EMA/ EU-Zulassung (= zwei Volldosen) könnte zu einer positiveren Wirksamkeit führen (so eine der Phase III-Studien); eine solche Anwendung wäre jedoch off-label-use.
Neuere Studien ${ }^{39}$ belegen eine Wirksamkeit schon nach der ersten Dosis insbesondere hinsichtlich schwerer Krankheitsverläufe.

Nebenwirkungen

etwa $75 \%$ der Geimpften haben leichte bis mäßige lokale, etwa $73 \%$ systemische UAW; grippeähnliche Erscheinungen, Fieber und Schüttelfrost treten häufiger auf als bei den mRNA-Impfstoffen ${ }^{40}$. Gegenanzeigen

Immunsupprimierte Personen, Schwangere und Jugendliche nicht in Prüfung eingeschlossen, sind bisher nicht untersucht; deshalb keine Impfung.

Es handelt sich um eine bedingte Zulassung der EU, bei der kein Unterschied bezüglich der Altersgruppen gemacht wurde; zugelassen ab 18 Jahre; Empfehlung der STIKO 18-64 Jahre; Altersbeschränkung durch CoronaImpfV v. 10.3.2021 aufgehoben.

5. Johnson\&Johnson ${ }^{41}$ (Vector-Impfstoff; Ad26. COV2.S, Zulassungsantrag (durch Janssen-Cilag) bei EMA gestellt; Liefervertrag mit EU; zur Phase III-Prüfung bisher nur Herstellerangaben)

Wirksamkeit

(relativ) 66\% Schutz vor einer moderaten bis schweren COVID-19-Infektion nach 14 Tagen; 85\% Schutz vor schweren COVID-19-Infektionen nach 28 Tagen; nur eine Impfdose; Wirksamkeit soll auch gegen südafrikanische Mutation bestehen.

Nebenwirkungen

niedriges bis mäßiges Niveau unerwünschter Wirkungen vergleichbar zwischen Verum- und PlaceboGruppe (bisherige Herstellerangabe).

Gegenanzeigen

bisher keine Angaben

Der Impfstoff erfordert nur eine Impfung und ist einfach lagerfähig.

33) Dazu Polack et al., Safety and efficacy of the BNT162b2 mRNA Covid-19 vaccine, N Engl J Med. 2020; 383: 2603-2615; Dagan et al., BNT162b2 mRNA Covid-19 Vaccine in a Nationwide Mass Vaccination Setting, N Engl J Med. February 24, 2021, DOI: 10.1056/NEJMoa2101765.

34) Auftreten von symptomatischen Infektionen ist bei Applikation des Verums gegenüber der Placebokontrollgruppe $95 \%$ geringer.

35) D.h. durch die Impfung auch die Ansteckung ausgeschlossen oder stark vermindert wird.

36) Dazu Baden et al., Efficacy and safety of the mRNA-1273 SARSCoV-2 vaccine. N Engl J Med. 2020 (published online Dec 30.), https://doi.org/10.1056/NEJMoa2035389.

37) Das Verfahren kann die Zulassung zeitlich beschleunigen, indem die Bewertung von Datenpaketen der pharmazeutischen und nichtklinischen Entwicklung bereits begonnen wird, bevor die vollständigen klinischen Daten für den formalen Antrag auf Zulassung vorliegen.

38) Überblick bei https://www.gesundheitsinformation.de/der-impfstoff-astrazeneca-azd1222-astrazeneca-zur-impfung-gegen-corona.html.

39) Hung/Poland, Single-dose Oxford-AstraZeneca COVID-19 vaccine followed by a 12-week booster, The Lancet, Volume 397 Number 10277, p 854-855.

40) PEI Sicherheitsbericht v. 4.3.2021, https://www.pei.de/ SharedDocs/Downloads/DE/newsroom/dossiers/sicherheitsberichte/sicherheitsbericht-27-12-bis-26-02-21.pdf? blob $=$ publicationFile $\& v=8$, Tabelle, S. 9 (zuletzt abgerufen 5.3.2021).

41) S. erste Informationen aus einer Phase I-IIa Studie: Jerald Sadoff u. a., Interim Results of a Phase 1-2a Trial of Ad26.COV2.S Covid-19 Vaccine, NJEM January 13, 2021 DOI: 10.1056/NEJMoa2034201 (zuletzt abgerufen 17.2.2021); die weiteren Daten beruhen auf einer Veröffentlichung von Zwischenergebnissen einer Phase 3-Studie durch Janssen, https://www.janssen.com/ germany/covid-19-impfstoffkandidat-zwischenergebnisse-derphase-3-studie (zuletzt abgerufen 17.2.2021). EMA empfiehlt Zulassung am 11.3.2021. 
6. Novavax ${ }^{42}$ (Protein-Subunit-Impfstoff; NVXCoV2373; noch kein Zulassungsantrag bei EMA gestellt, Liefervertrag mit EU)

Wirksamkeit

(relativ) $89,3 \%$ Schutz vor einer moderaten bis schweren Covid-19-Infektion nach 7 Tagen; zwei Impfdosen; soll gegen britische wie südafrikanische Mutation wirken; bisher nur Herstellerangaben)

Die drei Kriterien der Beschreibung der Impfstoffe sind aus der Sicht des Verf. die für die Aufklärung von zu Impfenden relevantesten ${ }^{43}$. Über ein weiteres entscheidungsrelevantes Kriterium, nämlich das der Immunogenität, gibt es bisher keine gesicherten Erkenntnisse. Eine durch die Impfung ausgelöste (sterile) Immunität wäre ein zusätzliches wichtiges Unterscheidungskriterium zwischen den Impfstoffen.

Nach einer neuen empirischen Studie über (auch) die Priorität der Impfstoffauswahl ${ }^{44}$ ergibt sich folgendes Bild:

\begin{abstract}
„Wer sich in Deutschland impfen lassen möchte, hat mit 48,2 Prozent keine Präferenz für einen der bislang zugelassenen Impfstoffe. Wenn eine Wahl des Vakzins möglich wäre, würden sich 33,3 Prozent für Biontech/Pfizer entscheiden, 5,8 Prozent für Moderna und nur zwei Prozent für AstraZeneca. Eine Verschiebung der zweiten Impfung, um zunächst mehr Menschen zu versorgen, befürworten hierzulande nur 19 Prozent der Befragten. 51 Prozent lehnen dies ab. In Großbritannien, wo dies bereits umgesetzt wird, stimmen 41 Prozent einer späteren Zweitimpfung zu."
\end{abstract}

Der Vergleich zwischen den genannten Impfstoffen ist unter Berücksichtigung der unterschiedlichen Datenlage auf die in der EU zugelassenen Impfstoffe konzentriert. Für andere Impfstoffe werden nur vorsichtige Hinweise gegeben.

\section{Knappheit, Priorisierung und Aufklärung}

Die Priorisierung nach der Corona-ImpfV ${ }^{45}$ ist eine Folge der Risikobewertung nach dem $\mathrm{Maß}$ der Gefährdung von Personengruppen durch COVID-1946. Sie ist weiter eine Folge der Impfstoffknappheit am Beginn der Impfungen. Eine Impfstoffknappheit kann sich bei nur einem oder bei mehreren zur Verfügung stehenden Impfstoffen äußern. An den allgemeinen Aufklärungsmodalitäten ändern diese unterschiedlichen Situationen nichts. Das hier im Mittelpunkt stehende Problem der Alternativenaufklärung stellt sich nur im zweiten Fall.

Im Februar 2021 stehen drei in der EU zugelassene Impfstoffe zur Verfügung: BioNTech/Pfizer, Moderna und AstraZeneca, wobei Letzterer gemäß der Empfehlung der STI$\mathrm{KO}$ (nur und entgegen der Zulassung durch die EMA) für die Altersgruppe 18-64 Jahren zur Verfügung gestellt wird, während die beiden anderen auch für die Altersgruppe $a b$ 65 Jahren in der Reihenfolge der STIKO-Empfehlung angeboten werden. Die Altersbegrenzung wurde am 10.3.2021 nach neueren Studien durch die neue CoronaImpfV mit Wirkung zum 8.3.2021 aufgehoben. Das Aufklärungsszenario stellt sich prinzipiell in allen Konstellationen für alle Personen in gleicher Weise. Wegen der übereinstimmenden Zulassungen der Impfstoffe wäre über alle gleichermaßen aufzuklären, auch wenn tatsächlich nicht alle für alle angeboten werden, denn die Entscheidung ob und mit welchem Impfstoff die zu impfende Person geimpft werden will, obliegt unter der Voraussetzung ,gleiche Zulassung“ alleine dieser Person (siehe genauer unten 7). Das ist die Kehrseite der fehlenden Impfpflicht - wobei hier dahingestellt bleiben soll, ob bei bestehender Impfpflicht die Impfung eines bestimmten Impfstoffes priorisiert angeordnet werden könnte ${ }^{47}$.

Die folgenden Ausführungen behandeln zunächst die Konstellation bis zum 8.3.2021 und anschließend die nach
Aufhebung der Altersbegrenzung für den AstraZenecaImpfstoff durch die CoronaImpfV v. 10.3.2021.

\section{Vergleichbare Nutzen: ärztliche Wahlfreiheit}

Wenn zwei oder mehrere Arzneimittel im identischen Indikationsgebiet mit gleicher Indikation und vergleichbaren Wirksamkeits- und Risikoprofilen nach kompetenter Nutzenbewertung durch die einschlägigen Institutionen konkurrieren, spricht man davon, dass sie gleichermaßen dem medizinischen Standard (vgl. $\$ 630$ a Abs. 2 BGB) entsprechen und deshalb die Ärztin/der Arzt eine Wahlfreiheit (,,echte Wahlmöglichkeit für den Arzt/die Ärztin“") zwischen ihnen für die Behandlung der Patienten hat ${ }^{48}$. Ärzte entscheiden über das auszuwählende und anzuwendende Arzneimittel. Das gilt ebenso für solche vergleichbaren Impfstoffe im selben Indikationsgebiet (= COVID-19). Bei gleicher Indikation (z.B. Alter), vergleichbarer Wirksamkeit (zwischen 90 und $95 \%$ ) und vergleichbaren unerwünschten Wirkungen (= Risiken, Nebenwirkungen, Wechselwirkungen), wie es bei den Impfstoffen von BioNTech/Pfizer und Moderna der Fall ist, entschieden also die Ärzte bzw. das zur Verfügung stehende Angebot und nicht die Patienten/zu Impfenden über den Einsatz des einen oder anderen Impfstoffs.

$\mathrm{Ob}$ das auch für den Impfstoff von AstraZeneca gilt, wäre wegen der altersbezogenen Bewertung der STIKO (nicht für Personen ab 65) mindestens zweifelhaft, auch wenn das gekennzeichnete Defizit (geringe Zahl der Älteren in den klinischen Studien der Phase III) in ähnlicher Weise für die anderen Impfstoffe gilt (dazu im Einzelnen unter 8). Nach Aufhebung der Altersbegrenzung wäre im Prinzip eine indikationsbezogene Vergleichbarkeit aller drei Impfstoffe gegeben, die dann allerdings im Hinblick auf unterschiedliche Wirksamkeit und unerwünschte Wirkungen zu differenzieren wäre und damit nicht mehr der ärztlichen Entscheidung unterläge.

\section{Unterschiedliche Nutzen: Patientenautonomie}

Handelt es sich um Arzneimittel/Impfstoffe mit unterschiedlichen Wirksamkeits- und Risikoprofilen bzw. unterschiedlichem Verhältnis zwischen Wirksamkeit und Risiken im identischen Indikationsgebiet, gelten andere Regeln. Der Diskussionsstand in Literatur und Rechtspre-

42) Information des pharmazeutischen Unternehmers Novavax v. 28.1.2021 über einen Zwischenbericht u.a. aus einer Phase IIIPrüfung des Impfstoffs: https://ir.novavax.com/news-releases/ news-release-details/novavax-covid-19-vaccine-demonstrates893-efficacy-uk-phase-3.

43) Für die Richtigkeit meiner Angaben habe ich mir bei Prof. Dr. med. W.-D. Ludwig, Vorsitzender der Arzneimittelkommission der deutschen Ärzteschaft (Akd ̈̈) fachlichen Rat eingeholt. Für seine Bereitschaft zum Gespräch danke ich herzlich. Für alle verbleibenden Fehler bin ich alleine verantwortlich.

44) Hamburg Center for Health Economics (HCHE), Ergebnisse der 5. Befragungswelle v. 19.1. - 1.2.2021 - COVID-19-Studie: Impfbereitschaft und Vertrauen in die Impfstoffe steigt, https:// www.hche.uni-hamburg.de/corona/5-fuenfte-befragungswelle.html (zuletzt abgerufen 17.2.2021)

45) \S 2-4 Corona-Impf V v. 8.2.2021, BAnz. AT 8.2.2021 V1.

46) STIKO-Empfehlung zur COVID-19-Impfung, Beschl. der STIKO zur 2. Aktualisierung der COVID-19-Impfempfehlung, Epidemiologisches Bulletin 5 | 2021, https://www.rki.de/DE/ Content/Infekt/EpidBull/Archiv/2021/Ausgaben/05_21.pdf? _blob=publicationFile (zuletzt abgerufen 9.2.2021).

47) S. auch Rixen, in: Katzenmeier (Hrsg.), FS f. Dieter Hart, Medizin - Recht - Wissenschaft, 2020, S. $483 \mathrm{ff}$.

48) Allgemein Katzenmeier, in: Laufs/Katzenmeier/Lipp, Arztrecht, Kap. V, S. 133 ff.; Deutsch/Spickhoff, Medizinrecht, 7. Aufl. 2014, Rdnrn. 438 ff.; Pauge/Offenloch, Arzthaftungsrecht - Neue Entwicklungslinien der BGH-Rechtsprechung, 14. Aufl. 2018, Rdnrn. $403 \mathrm{ff}$. 
chung über diese sog. Alternativenaufklärung ${ }^{49}$ ist, wie folgt, bei Hart, Aufklärung bei der Arzneimittelbehandlung ${ }^{50}$ dargestellt:

„Die Aufklärung über unterschiedliche verfügbare und gleichermaßen indizierte Behandlungsalternativen (,echte Wahlmöglichkeit") ist Teil beider Formen der Selbstbestimmungsaufklärung - also der Verlaufs- und Risikoaufklärung ${ }^{51}$. Sie hat - auch im Sinne einer gemeinsamen Entscheidungsfindung zwischen Patient und Arzt ${ }^{52}$ - zu erfolgen, wenn solche Behandlungen mit unterschiedlichen Nutzen, also Chancen und Risiken für den Patienten verbunden sind. Die Pflicht zur Alternativenaufklärung gilt ebenso für Arzneimittelbehandlungen, also sowohl für unterschiedliche Arzneimittel wie für Arzneimittel zu sonstigen Behandlungen. Eine Korrelation von gemeinsamer Entscheidungsfindung und Alternativenaufklärung ist insbesondere bei der Behandlung von Erkrankungen im onkologischen Formenkreis angesagt, weil sich hier häufig unterschiedliche Arzneimitteltherapien bzw. Therapiestrategien mit unterschiedlichen Nutzen/Risiko-Profilen gegenüberstehen. Insofern ist die Onkologie geradezu das Bewährungsfeld guter Alternativenaufklärung als Voraussetzung einer selbstbestimmten Patientenentscheidung und gerade nicht das Feld ärztlicher Wahlfreiheit. Unterschiedliche gleichermaßen indizierte und verfügbare Chemotherapien bzw. Therapiekombinationen beispielsweise erfordern sowohl eine abstrakt-generelle (Produktbezug) als auch eine situativ-individuelle (Behandlungsbezug) Aufklärung, die nicht - sofern medizinisch möglich Behandlungsdruck, sondern Entscheidungschancen für den Patienten aufbaut. Dabei ist auch über die vorhandene Evidenz für die in Betracht kommenden Behandlungen vergleichend $\mathrm{zu}$ informieren. Entscheidend bei konkurrierenden Arzneimittelbehandlungen sind insbesondere unterschiedliche Nutzen-, Wirksamkeits- und Risikoprofile (unterschiedliche Wirkstoffe oder Kombinationen von Wirkstoffen). Solche Alternativen sind im Rahmen der Risikoaufklärung dem $\mathrm{Pa}$ tienten verständlich darzulegen, sofern es sich um Nutzen- oder/und Risikounterschiede handelt, die als Arzneimittelwirkungen nach den genannten Grundsätzen aufklärungsbedürftig wären. Beispielsweise ist bei der Aufklärung über die Anwendung von Kontrazeptiva deren jeweiliges Risikoprofil bei der Information von Frauen unterschiedlicher Risikogruppen zu beachten ${ }^{53}$. Die Grundsätze der Aufklärung über Behandlungsalternativen gelten auch dann, wenn die indizierte Alternative in der gesetzlichen Krankenversicherung nicht zum Leistungsumfang gehört ${ }^{54}$."55

Überträgt man diese Grundsätze auf die Situation konkurrierender Impfstoffe gegen COVID-19, so ergibt sich Folgendes:

1. Die Typen der Impf(wirk)stoffe sind zu unterscheiden, wenn sie hinsichtlich der Indikation zu differenzieren sind: z. B. Alter.

2. Die unterschiedlichen Typen der Impf(wirk)stoffe sind zu unterscheiden, wenn mit den unterschiedlichen Wirkstoffen unterschiedliche Nutzen (Wirksamkeit und Nebenwirkungen und ihr Verhältnis) verbunden sein können.

3. Innerhalb der Wirkstoffgruppen können, müssen aber nicht unterschiedliche Nutzen bestehen.
Diese Differenzierungen werden anschließend auf die verschiedenen in der EU zugelassenen Impfstoffe bezogen.

\section{Zwischen ärztlicher Wahlfreiheit und Patienten- Selbstbestimmung: Impfstoffe im Vergleich}

\subsection{Indikationsdifferenzierungen schließen echte Wahlmöglichkeit aus}

Allein auf die Zulassung der Impfstoffe bezogen hätten wir es bei BioNTech, Moderna und AstraZeneca mit echten Wahlmöglichkeiten zu tun. Da aber die Indikationsdifferenzierung der STIKO, AstraZeneca nur 18-64 Jahre, in $₫ 2$ Abs. 2 CoronaImpfV (alt) übernommen wurde, bestehen unterschiedliche altersbezogene Indikationen, so dass eine echte Wahlmöglichkeit $=$ identischer Standard der Impfbehandlung ausgeschlossen ist (soweit Personen über 65 betroffen sind). Seit der Aufhebung der Altersdifferenzierung durch CoronaImpfV (neu) wäre im Prinzip eine echte Wahlmöglichkeit für die Ärzte gegeben.

Zwischen BioNTech/Pfizer und Moderna dagegen besteht wegen der Nutzen-, Wirksamkeits- und Nebenwirkungsähnlichkeit auf der Basis der Bewertungen aller einschlägigen Institutionen Vergleichbarkeit ${ }^{56}$ und damit eine Wahlmöglichkeit und eine ärztliche Entscheidungsfreiheit zwischen den Impfstoffen. Der im Rahmen des zur Verfügung stehenden Angebots vorhandene Impfstoff wird dem zu Impfenden appliziert.

Die Indikationskonkurrenz zwischen den Impfstoffen BioNTech/Pfizer, Moderna und AstraZeneca in den Impfgruppen unter 18 (16-18 nur BioNTech/Pfizer; keine Konkurrenz) und über 64 Jahren (BioNTech/Pfizer und Moderna) ist ebenso zu behandeln. Seit Aufhebung der Altersbegrenzung für den AstraZeneca-Impfstoff steht die Vergleichbarkeit insbesondere hinsichtlich der Wirksamkeit und der Nebenwirkungen in Frage. Nach den Sicherheitsberichten des $\mathrm{PEI}^{57}$ treten jedenfalls hinsichtlich der Nebenwirkungen grippeähnlicher Erscheinungen, Fieber und Schüttelfrost erhebliche Häufigkeitsunterschiede auf, die den Ausschluss der echten Wahlmöglichkeit und eine Wahlmöglichkeit der zu Impfenden - möglicherweise auch

49) Katzenmeier, in: Laufs/Katzenmeier/Lipp, Arztrecht, Kap. V, S. $133 \mathrm{ff}$.

50) Hart, in: Rieger/Dahm/Katzenmeier/Stellpflug/Ziegler (Hrsg.), HK-AKM, Stichwort Nr. 643, Rdnrn. 34; ders., Arzneimittelbehandlung, ebd., Stichwort Nr. 240, Rdnrn. $21 \mathrm{ff}$.

51) BGH, MedR 2005, S. 599 - Behandlung einer Fehlstellung nach Bruch des Handgelenks; grundlegend BGHZ 102, $17=$ NJW 1988, 763 = JZ 1988, 411 - Elektrokoagulation; dazu Damm, NJW 1989, S 737 ff.; Katzenmeier, in: Laufs/Katzenmeier/Lipp, Arztrecht, Kap V, S. 133 ff.; vgl. Hager, in: Staudinger, BGB, 2009, $\$ 823$, Rdnrn I $92 \mathrm{ff}$; Wagner, in: MüKo/BGB, 8. Aufl. 2020 , BGB $\$ 630$ e, Rdnrn. 21-24.; Wenzel, in: Wenzel (Hrsg.), Medizinrecht, 3. Aufl. 2013, Kap 4, A, Rdnrn. $119 \mathrm{ff}$.

52) S. zu Behandlungsalternativen und gemeinsamer Entscheidungsfindung Härter u. a., DÄBl. Int. 2015; 112(40): 672-9, der Aufsatz thematisiert Behandlungsprozesse in der Onkologie, in denen häufig Arzneimittelalternativen zur Entscheidung anstehen.

53) Vgl. Hart, MedR 2003, 603, 607.

54) Stöhr, MedR 2010, 214, 217 (auch unter dem Aspekt wirtschaftlicher Aufklärung).

55) So auch RKI Impfstofftypen, https://www.rki.de/SharedDocs/ FAQ/COVID-Impfen/gesamt.html (zuletzt abgerufen 16.2.2021).

56) Überblick bei PEI, https://www.pei.de/DE/arzneimittel/impfstoffe $/$ covid-19/covid-19-node.html;jsessionid=0DB7402C454 5267B2D1F8102658D6858.intranet221 (zuletzt abgerufen am 15.3.2021)

57) Zuletzt Sicherheitsbericht vom 4.3.2021 bis zum 26.2.2021, https://www.pei.de/SharedDocs/Downloads/DE/newsroom/ dossiers/sicherheitsberichte/sicherheitsbericht-27-12-bis-26-0221.pdf?__blob=publicationFile $\& v=85$, insbesondere Tabelle S. 9 (zuletzt abgerufen am 5.3.2021). 
differenziert nach Vorerkrankungen und/oder Alter - nahelegen (8.2).

Sollten zu Impfende zwischen den beiden bzw. drei Impfstoffen auswählen wollen, bleibt ihnen nur die Ablehnung des angebotenen und die Suche nach dem Angebot des anderen evtl. an einem anderen Ort. Der Anspruch auf Impfung nach $\$ 1$ CoronaImpfV wird durch diese Ablehnung nicht ausgeschlossen, weil nicht die Impfung an sich, sondern nur die Impfung mit einem bestimmten Impfstoff abgelehnt wird und Sinneswandlungen durchaus möglich und nicht sanktioniert sind.

Unklar bleibt, ob und wieweit die unterschiedlichen Lagerungseigenschaften eine echte Wahlmöglichkeit negativ beeinflussen. Wird die Impfung auch in Praxen der Vertragsärzte ermöglicht, könnte der BioNTech/Pfizer-Impfstoff wegen seiner Kühlungsnotwendigkeit $\left(-70 \mathrm{Grad}^{58}\right)$ aus der Anwendung dort ausscheiden.

\subsection{Unterschiedliche Nutzen schließen ärztliche Wahlmöglichkeit aus}

Jenseits des Indikationsbezugs sind Behandlungen unterschiedlichen Nutzens die Bewährung der Selbstbestimmungsaufklärung und der Patientenentscheidung. In der Konkurrenz zwischen entweder BioNtech/Pfizer, Moderna und AstraZeneca: Ihre unterschiedlichen Wirksamkeitseigenschaften (etwa 90-95\% zu 70\%; Nebenwirkungshäufigkeiten) schließen eine echte Wahlmöglichkeit aus und erfordern eine Aufklärung über die unterschiedlichen Eigenschaften und eine selbstbestimmte Entscheidung der zu Impfenden. Wenn Indikations- und Wirksamkeits- und/oder Risikounterschiede zusammenkommen und deshalb AstraZeneca innerhalb der Priorisierungsgruppe 1 indikationsbezogen nur für das Risikopersonal nach $₫ 2$ Abs. 1 S. 1 Nr. 2-5 CoronaImpfV (alt) in Frage kommt, gleichzeitig aber dieser Impfstoff mit BioNTech/Pfizer und Moderna konkurriert, steht dieser Personengruppe der Beschäftigten mit höchstem Risikopotenzial die Entscheidung zwischen den Impfstoffen zu. Sie könnten vertragsrechtlich nach Aufklärung auswählen. Dasselbe gilt insgesamt nach Aufhebung der Altersbegrenzung für die drei Impfstoffe.

Diese Auswahlmöglichkeit wird ihnen durch $₫ 1$ Abs. 2 CoronaImpfV zwar nicht genommen, aber jedenfalls zeitweise versagt, weil , diese Personen jedenfalls vorrangig mit diesen Impfstoffen versorgt werden" sollen. Solange das Impfstoffangebot insgesamt für die Priorisierungsgruppe 1 nicht ausreicht, aber der eine indikationsbezogene Impfstoff für die Jüngeren ,,verimpft“ werden könnte, soll dieser Impfstoff bei den jüngeren Personen der Priorisierungsgruppe 1 ( $\$ 2$ Abs. $2 \mathrm{~S}$. 1 CoronaImpfV alt) angewendet werden. Sollte eine Person den wirksameren/nebenwirkungsärmeren mRNA-Impfstoff wünschen, bleibt nur die „Warteliste“. Die CoronaImpfV beschränkt nicht die Aufklärung, aber die eigentlich daraus folgende Auswahlmöglichkeit zeitlich.

Entfällt diese zeitweise Beschränkung besteht die Konkurrenz unterschiedlicher Impfstoffe mit Aufklärungs- und Auswahlkonsequenzen für den Patienten/zu Impfenden.

\section{3 Ärztliche Entscheidung trotz unterschiedlicher Nutzen ${ }^{59}$ ?}

Im Falle von erheblichen Vorbelastungen (z. B. bekannte Neigung zum anaphylaktischen Schock) älterer Personen der Gruppe 1 kann es dazu kommen, dass die/der Impfärztin/arzt die Impfung beispielsweise mit dem (vorhandenen) BioN tech/Pfizer-Impfstoff mangels Indikation bzw. wegen Gegenanzeigen ablehnt und den von AstraZeneca impfen will - dort fehlt der parallele Hinweis auf eine Gegen- anzeige $^{60}-$, der aber für den 80 jährigen mit Vorerkrankung aufgrund der STIKO-Empfehlung nicht geeignet erscheint. Das ist kein Fall eines off-label-use, weil eine Applikation mit der Zulassung durch die EMA/EU übereinstimmt. Rechtlich ist das ein Fall vorbehaltener ärztlicher Entscheidung; es gibt kraft medizinischer Indikation (bisher) keine Alternative. Das Problem ist nach Aufhebung der Altersbegrenzung entfallen.

Häufiger sind die Fälle, in denen Personen der ersten Gruppe bei vorhandenem Angebot von AstraZeneca und einem $m R N A$ Impfstoff den $m R N A$-Impfstoff wünschen und den nach $\$ 2$ Abs. 2 S. 1 CoronaImpfV vorgesehenen AstraZeneca-Impfstoff wegen der geringeren Wirksamkeit bzw. der Nebenwirkungsdifferenz ablehnen. Da der mRNA-Impfstoff insbesondere für die über 80jährigen vorgehalten wird, lehnen Impfärzte „im Rahmen der Verfügbarkeit“ den Wunsch nach dem mRNA-Impfstoff $a b$, weil damit die Planbarkeit bzw. Planung der Impftermine nicht mehr eingehalten werden könnte. Es handelt sich eigentlich um einen Fall der Konkurrenz unterschiedlicher Impfstoffeigenschaften und damit der Selbstbestimmung der zu Impfenden. Die Beschränkung folgt m. E. aus $\$ 1$ Abs. 1 CoronaImpfV: Der Rahmen der zur Verfügung stehenden Impfstoffe ermächtigt die ablehnende ärztliche Entscheidung. Auch diese bisher bestehende Fallgruppe ist nur noch hinsichtlich der Nutzendifferenzierung zwischen den mRNA-Impfstoffen und dem von AstraZeneca gegeben.

\section{Resümee}

Die individuelle Aufklärung der zu Impfenden ist ein wichtiger Prozess und Teil der notwendigen allgemeinen Information und Beratung über die Impfstoffe. Aufklärung ist ein wichtiger Teil der Legitimation der Impfung und ihrer Praxis. Gute Aufklärung ist ,partizipative Kommunikation“. Sowohl Sicherungs- wie Selbstbestimmungsaufklärung erfordern eine ,evidenzbasierte Information“ auf der Basis der aktuellen Erkenntnisse über die anwendbaren Impfstoffe. Dabei entscheiden IfSG, SGB V und die CoronaImpf $\mathrm{V}$ über den Rahmen, innerhalb dessen Impfstoffe zur Impfung zur Verfügung stehen. Die CoronaImpfV schließt entgegen ihrer Begründung mögliche Wahlrechte der zu Impfenden nicht allgemein, sondern nur unter bestimmten Voraussetzungen der Knappheit und Priorisierung aus. Jenseits dieses begrenzt wirksamen Ausschlusses besteht ein ärztliches Wahlrecht, wenn die Impfstoffe eine echte Wahlmöglichkeit darstellen, also bei identischer Indikation vergleichbaren Nutzen haben und damit standardgemäß sind. Wahlrechte der zu Impfenden bestehen bei identischer Indikation, aber unterschiedlichen Nutzen. Fehlt - auch innerhalb einer priorisierten Gruppe - der berechtigterweise gewählte Impfstoff, führt das nicht zum Verlust des Impfanspruchs, sondern nur zu einer zeitlichen Verlagerung der Impfung.

Die IfSG/SGB V/CoronaImpfV begrenzen in ihrer gegenwärtigen Fassung die Patientenselbstbestimmung über die Auswahl zwischen Impfstoffen unterschiedlicher Nutzen. Beim gegenwärtigen Stand der Entwicklung der Impfstoffe und der Kenntnis über ihre Eigenschaften ist diese Begrenzung nicht akzeptabel.

58) Nach neueren Informationen soll der Impfstoff auch bei Temperaturen zwischen minus 8-15 Grad länger lagerfähig sein.

59) Hinweise auf diese Konstellationen aus der Praxis der Impfung verdanke ich Dr. med. M. Gruhl, der als Impfarzt tätig ist.

60) Dazu während der Fahnenkorrektur EMA zu thrombotischen Ereignissen, https://www.ema.europa.eu/en/news/covid-19-vaccine-astrazeneca-prac-investigating-cases-thromboembolic-eventsvaccines-benefits (zuletzt abgerufen 12.3.2021). 